\title{
Assessment of Treatment Procedures by Means of Bacteriological and Histological Examinations
}

\author{
M. ROSENFELD
}

Forschungsinstitut Borstel, 2061 Borstel, West Germany

The decisive criterion for assessing the value of a therapy directed towards a high antibacterial effect, must be its action on the bacteria. We use biopsies taken at monthly intervals as random sample of the patient's bacterial status. Bacillary counting is made by simultaneous examinations. As it is our aim to obtain reduction of the number of bacteria to zero, any detectable acid-fast material, whether solid, granulated bacilli or bacterial residues, is classified as "positive".

(1) The best way of determining the quantitative decrease in the number of bacteria during therapy is by counting. We therefore apply the technique after Rees, modified by Krenzien, i.e. mycobacterial counting in the tissue homogenate of formalin-fixed biopsies. The material is stained with carbol fuchsin by heating three times. We differentiate with $4 \%$ sulphuric acid for exactly 1 minute and counterstain with malachite green. The lower measuring limit of this method is approximately $10^{2}$ organisms $/ \mathrm{mg}$ biopsy when the homogenate is not further diluted, and counting is performed according to the aforementioned method.

(2) A further basis for assessing the therapeutic value is the examination of frozen sections. The sections are prepared from the same biopsy, the other part of which has been homogenized for counting. Cold staining of the section with carbol fuchsin after Pattyn reveals the best results. We differentiate with $0.1 \%$ hydrochloric alcohol or with $2-4 \%$ sulphuric acid and counterstain with malachite green or methylene blue. This technique allows an exact examination of the entire section with regard to the number of bacteria in nerves, muscles, vessels, etc. and the diagnosis of the histopathological situation. We consider this method as the most important for the decision "negative" or "positive".

(3) Smears are collected together with the biopsies. In accordance with the Havel technique, blotting paper with carbol fuchsin is placed on the smear and heated only once; differentiation with $0.1 \%$ hydrochloric alcohol. This is a very careful technique to harvest a high number of bacteria.

The results obtained by means of these 3 methods are compared with one another for control and final evaluation. As we know from our experimental work with different mycobacteria in different macroorganisms that dead bacteria are also able to cause lengthy granulomatous processes after injection into the tissue and that the bacilli themselves can stay acid-fast, even solidly acid-fast, over a 
longer period (granulation is a reactional stage of living mycobacteria), the decrease of mycobacterial mass per $\mathrm{mg} /$ biopsy is an important indication to the value and efficacy of a therapeutic measure. The elimination of dead material is therefore included in this criterion for the assessment of a treatment.

This method appears to be satisfactory as far as practical work is concerned. We must, however, bear in mind that mycobacteria not only vary tremendously in shape and structure, but that they are even able to lose their acid-fastness under suitable conditions and nonetheless can multiply and become acid-fast again.

We are certainly aware of the fact that the described method represents no optimal solution. But it quite reliably indicates the trend, despite occasional discrepancies between the results of the different techniques. The examples in the tables may verify this opinion.

Tables 1-3 follow on pages 55-58. 
TABLE 1

A case after 2 years of treatment in the status to be discussed as "negative". Patient is not yet classified as "negative". Twenty years of pre-treatment with dapsone
Malta
30
B. J.
40
SB009
Serial No.
Name
Age
Pat. No.

\begin{tabular}{|c|c|c|c|c|c|c|c|c|}
\hline \multirow{2}{*}{ Date } & \multicolumn{2}{|c|}{ Skin-smear } & \multicolumn{2}{|c|}{ Biopsy } & \multicolumn{4}{|c|}{ Histology } \\
\hline & ${ }^{\mathrm{a}} \mathrm{Ma}$ & ${ }^{b}$ Bo & HBI & HMI & Dil. & Bact. & Remarks & Therapy \\
\hline 1972 & & & & & & & & \\
\hline July 21 & + & & & & & ++ & & \\
\hline August & & & & & & & & \\
\hline Sept. $2 / 30$ & & ++ & $5.20 \times 10^{5}$ & $16 / 100$ & $1: 2$ & +++ & & \\
\hline & & ++ & $1.09 \times 10^{6}$ & $10 / 100$ & $1: 2$ & +++ & & \\
\hline Oct. 28 & ++ & ++ & $5.58 \times 10^{4}$ & $0 / 28$ & $1: 2$ & & & \\
\hline Nov. & ++ & +++ & & & & & & \\
\hline Dec. 9 & + & + & $6.78 \times 10^{4}$ & $0 / 67$ & $1: 2$ & & & \\
\hline 1973 & & & & & & & & \\
\hline Jan. 8 & ++ & +++ & $1.48 \times 10^{3}$ & $0 / 20$ & $1: 2$ & ++ & & \\
\hline Feb. 3 & ++ & +++ & $1.34 \times 10^{4}$ & $0 / 4$ & $1: 2$ & +++ & & \\
\hline $\operatorname{March} 3 / 31$ & + & & $1.54 \times 10^{4}$ & $0 / 8$ & $1: 2$ & & & \\
\hline & + & & $1.87 \times 10^{4}$ & $0 / 13$ & $\emptyset$ & & & \\
\hline April 29 & & & $5.27 \times 10^{3}$ & $0 / 11$ & $\emptyset$ & & & \\
\hline May 26 & + & + & $1.28 \times 10^{5}$ & $1 / 93$ & $\emptyset$ & +++ & & \\
\hline June 23 & - & & $2.60 \times 10^{4}$ & $1 / 40$ & $\emptyset$ & & & RAMP \\
\hline July 21 & - & & & & & ++ & & \\
\hline August 18 & - & & $9.68 \times 10^{2}$ & $1 / 12$ & $\emptyset^{d}$ & + & & \\
\hline Sept. 15 & $+\mathrm{sc}$ & & $2.96 \times 10^{3}$ & $1 / 40$ & $\emptyset^{d}$ & ++ & & \\
\hline Oct. 13 & ++ & ++ & $1.08 \times 10^{5}$ & $4 / 38$ & $1: 2$ & +++ & & \\
\hline Nov. 10 & + & + & $1.67 \times 10^{3}$ & $0 / 16$ & $\emptyset^{d}$ & +++ & & \\
\hline Dec. 15 & + & + & $3.10 \times 10^{2}$ & $0 / 3$ & $\emptyset^{d}$ & ++ & & \\
\hline 1974 & & & & & & & & \\
\hline Jan. 12 & $+\mathrm{sc}$ & & $4.04 \times 10^{4}$ & $0 / 25$ & $\emptyset$ & ++ & & \\
\hline Feb. 9 & & + & $2.45 \times 10^{2}$ & $0 / 2$ & $\emptyset^{d}$ & ++ & & \\
\hline March 9 & - & + & $1.61 \times 10^{4}$ & $0 / 20$ & $\emptyset$ & & & \\
\hline April 6 & - & $(+)$ & $7.40 \times 10^{1}$ & $1 / 1$ & $\theta^{d}$ & $((+))$ & & \\
\hline May 4 & - & & & & & $((+))$ & & \\
\hline June 1 & - & & $3.05 \times 10^{2}$ & $0 / 1$ & $\emptyset^{d}$ & + & & \\
\hline July $1 / 27$ & $-1-$ & & $\emptyset$ & $\emptyset$ & $\emptyset^{d}$ & $\emptyset$ & & \\
\hline August 24 & - & + & $\emptyset$ & $\emptyset$ & $\emptyset^{d}$ & $\emptyset$ & & \\
\hline Sept. 21 & & & $2.16 \times 10^{2}$ & $0 / 1$ & $\emptyset^{d}$ & $((+))$ & & \\
\hline
\end{tabular}

a Malta

b Borstel

sc scanty 
TABLE 2

$A$ case that will require even longer than 2 years. Fourteen years of pre-treatment
Malta
163
R. J.
Age
01864
Serial No.
Name
Age Pat. No.

\begin{tabular}{|c|c|c|c|c|c|c|c|c|}
\hline \multirow{2}{*}{ Date } & \multicolumn{2}{|c|}{ Skin-smear } & \multicolumn{3}{|c|}{ Biopsy } & \multicolumn{2}{|c|}{ Histology } & \multirow[b]{2}{*}{ Therapy } \\
\hline & ${ }^{\mathrm{a}} \mathrm{Ma}$ & ${ }^{\mathrm{b}} \mathrm{Bo}$ & $\mathrm{HBI}$ & HMI & Dil. & Bact. & Remarks & \\
\hline 1972 & & & & & & & & \\
\hline July & & & & & & $(+)$ & & \\
\hline August 4 & & ++ & & & $1: 2$ & +++ & & \\
\hline Sept. $1 / 29$ & $\begin{array}{c}++ \\
+++\end{array}$ & & $\begin{array}{l}2.82 \times 10^{5} \\
3.28 \times 10^{4}\end{array}$ & $\begin{array}{l}0 / 67 \\
0 / 12\end{array}$ & $\begin{array}{l}1: 2 \\
1: 2\end{array}$ & & & \\
\hline Oct. & & & & & & & & \\
\hline Nov. 3/21 & & +++ & $\begin{array}{l}3.09 \times 10^{5} \\
5.48 \times 10^{3}\end{array}$ & $\begin{array}{l}1 / 104 \\
0 / 25\end{array}$ & $1: \phi^{d}$ & ++ & & \\
\hline Dec. 19 & + & ++ & $1.12 \times 10^{5}$ & $0 / 75$ & $1: 2$ & & & \\
\hline $\begin{array}{l}1973 \\
\text { Jan. } 16 \\
\text { Feb. }\end{array}$ & ++ & $(+)$ & $3.59 \times 10^{3}$ & $0 / 5$ & $1: 2$ & + & & \\
\hline March 1 & & & $7.13 \times 10^{3}$ & $0 / 4$ & $1: 2$ & & & \\
\hline April 3 & + & & $2.94 \times 10^{3}$ & $0 / 3$ & $\emptyset$ & & & \\
\hline May $2 / 30$ & $\ddot{+}$ & $((+))$ & $\begin{array}{l}5.32 \times 10^{2} \\
2.52 \times 10^{4}\end{array}$ & $\begin{array}{l}0 / 1 \\
0 / 19\end{array}$ & $\begin{array}{c}\emptyset \\
1: 2\end{array}$ & + & & \\
\hline June 6 & + & & $1.00 \times 10^{4}$ & $0 / 18$ & $\emptyset$ & & & RAMP \\
\hline July & + & $(+)$ & & & & & & COMB. \\
\hline August & $+\mathrm{sc}$ & & & & & & & \\
\hline Sept. 18 & + & & $4.68 \times 10^{4}$ & $0 / 20$ & $1: 2$ & +++ & & \\
\hline Oct. 30 & + & & $3.05 \times 10^{3}$ & $0 / 21$ & $\emptyset^{d}$ & ++ & & \\
\hline Nov. 27 & + & & $5.48 \times 10^{3}$ & $0 / 25$ & $\emptyset^{d}$ & & & \\
\hline Dec. & & & & & & & & \\
\hline $\begin{array}{r}1974 \\
\text { Jan. } 8\end{array}$ & + & & $9.38 \times 10^{2}$ & $0 / 9$ & $\emptyset^{d}$ & & & \\
\hline Feb. 5 & - & $\emptyset$ & $2.08 \times 10^{2}$ & $0 / 3$ & $\emptyset^{d}$ & $(+)$ & & \\
\hline March 5 & + & + & $1.51 \times 10^{4}$ & $0 / 70$ & $\emptyset^{d}$ & $((+))$ & & \\
\hline April 19 & - & & & & & & & \\
\hline May 14 & - & & $1.91 \times 10^{3}$ & $1 / 3$ & $\emptyset^{d}$ & $\emptyset$ & & \\
\hline June 11 & + & & $\emptyset$ & $\emptyset$ & $\emptyset^{d}$ & $((+))$ & & \\
\hline July 9 & - & & $6.79 \times 10^{2}$ & $0 / 5$ & $\emptyset^{d}$ & $(+)$ & & \\
\hline August 6 & $+\mathrm{sc}$ & $(+)$ & $4.17 \times 10^{3}$ & $0 / 12$ & $\emptyset^{d}$ & $(+)$ & & \\
\hline Sept. 3 & - & & $9.62 \times 10^{2}$ & $0 / 3$ & $\emptyset^{d}$ & $((+))$ & & \\
\hline
\end{tabular}

a Malta

b Borstel

sc scanty 
TABLE 3

A case without pre-treatment, reaching the status to be discussed as "negative" already after 1 year

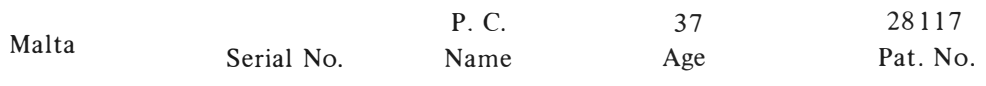

\begin{tabular}{|c|c|c|c|c|c|c|c|c|}
\hline \multirow{2}{*}{ Date } & \multicolumn{2}{|c|}{ Skin-smear } & \multicolumn{2}{|c|}{ Biopsy } & \multicolumn{4}{|c|}{ Histology } \\
\hline & ${ }^{\mathrm{a}} \mathrm{Ma}$ & ${ }^{\mathrm{b}} \mathrm{Bo}$ & HBI & HMI & Dil. & Bact. & Remarks & Therapy \\
\hline $\begin{array}{l}1973 \\
\text { July } \\
\text { August }\end{array}$ & & & & & & & & \\
\hline Sept. & +++ & & & & & & & \\
\hline Oct. 15 & + & & $1.12 \times 10^{5}$ & $1 / 44$ & $\emptyset$ & + & & \\
\hline Nov. 12 & ++ & ++ & & & & + & & \\
\hline $\begin{array}{l}\text { Dec. } 10 \\
1974\end{array}$ & + & & $3.48 \times 10^{4}$ & $5 / 44$ & $\emptyset$ & + & & \\
\hline $\begin{array}{l}\text { Jan. } 7 \\
\text { Feb. } 4\end{array}$ & $\begin{array}{l}+ \\
-\end{array}$ & & $\begin{array}{c}1.50 \times 10^{4} \\
\emptyset\end{array}$ & $\begin{array}{c}4 / 30 \\
\emptyset\end{array}$ & $\begin{array}{l}\emptyset^{d} \\
\emptyset^{d}\end{array}$ & $\begin{array}{l}+ \\
+\end{array}$ & & $\begin{array}{l}\text { RAMP } \\
\text { COMB. }\end{array}$ \\
\hline March 4 & + & ++ & $1.58 \times 10^{5}$ & $3 / 77$ & $1: 2$ & +++ & & \\
\hline April 1/29 & $+\mathrm{sc} /-$ & $(+)$ & $\begin{array}{l}2.03 \times 10^{3} \\
2.05 \times 10^{3}\end{array}$ & $\begin{array}{l}0 / 20 \\
3 / 20\end{array}$ & $\emptyset^{d}$ & $\begin{array}{l}((+)) \\
((+))\end{array}$ & & \\
\hline May 27 & -- & & $4.87 \times 10^{2}$ & $0 / 3$ & $\emptyset^{d}$ & $((+))$ & & \\
\hline June 24 & - & & $2.12 \times 10^{2}$ & $0 / 2$ & $\emptyset^{d}$ & $((+))$ & & \\
\hline July 22 & & & $5.21 \times 10^{2}$ & $0 / 2$ & $\emptyset^{d}$ & $((+))$ & & \\
\hline August 19 . & + & $((+))$ & $\emptyset$ & $\emptyset$ & $\emptyset^{d}$ & $((+))$ & & \\
\hline Sept. 16 & - & & $5 \times 10^{1}$ & $0 / 1$ & $\emptyset^{d}$ & $((+))$ & & \\
\hline
\end{tabular}

a Malta

b Borstel

sc scanty 
TABLE 4

A typical case with a lower number of bacteria. The reduction of the number of bacteria to zero is reached much more rapidly

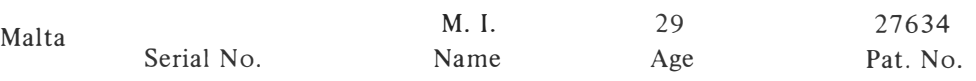

\begin{tabular}{|c|c|c|c|c|c|c|c|c|}
\hline \multirow{2}{*}{ Date } & \multicolumn{2}{|c|}{ Skin-smear } & \multicolumn{2}{|c|}{ Biopsy } & \multicolumn{4}{|c|}{ Histology } \\
\hline & $\mathrm{Ma}$ & Bo & HBI & HMI & Dil. & Bact. & Remarks & Therapy \\
\hline
\end{tabular}

1972

July

August

Sept.

Oct.

Nov.

Dec.

1973

Jan.

Feb.

March

April

May

June

July

August 6

Sept. 24

Oct.

Nov. 6

Dec. 16

1974

Jan. 15

Feb.

March 19

$+\quad 5.29 \times 10^{3}$

$5 / 44$

$\begin{array}{ll}\emptyset^{d} & ++ \\ \emptyset^{d} & \end{array}$

(+) $1.42 \times 10^{2} \quad 0 / 1 \quad \emptyset^{d}$

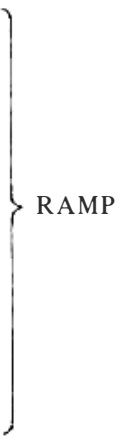

April

May

June

$\begin{array}{llll}\emptyset & \emptyset & \emptyset^{d} & + \\ \emptyset & \emptyset & \emptyset^{d} & \emptyset \\ \emptyset & \emptyset & \emptyset^{d} & \emptyset\end{array}$

$\emptyset \quad \emptyset \quad \emptyset^{d} \quad \emptyset$

June 